\title{
Weaving a Proper Net to Catch Large Objects in Wireless Sensor Networks
}

\author{
Alina Olteanu, Yang Xiao, Senior Member, IEEE, Kui Wu, Senior Member, IEEE, \\ and Xiaojiang Du, Senior Member, IEEE
}

\begin{abstract}
Wireless sensor networks consist of a large number of sensors and have been broadly used for intrusion detection in surveillance systems. To guarantee detection quality, such networks are usually over-engineered, i.e., more than required sensors are deployed and remain active in order to cover each point in the monitored field with a high probability at any time instance. Existing sensor scheduling schemes based on the point coverage model tightly weave a sensor "net" that is unnecessarily dense. Intuitively, when the size and the shape of intrusion objects are considered, any net with holes no smaller than the size of the intrusion object would work fine. With this design philosophy in mind, we build a new mathematical model to investigate the impact of size and shape of intrusion objects on network configuration. We derive analytical results that provide practitioners with insights on how to weave an effective sensor "net" for intrusion object detection with minimum number of active sensors.
\end{abstract}

Index Terms-Sensor networks, energy saving, intrusions, intrusion objects, optimization.

\section{INTRODUCTION}

$\mathbf{T}$ HE integration of sensor technology, wireless communication, and ad hoc networking leads to a popular network system, termed wireless sensor networks. Such networks have found various applications in civil monitoring and military surveillance, including for instance environment monitoring, intelligent transportation, smart home, and intrusion detection. In general, a large number of wireless sensor nodes are deployed in the field and report events (e.g., approaching of enemy tanks) to a processing center. Wireless sensor nodes are normally powered by battery and thus their energy consumption becomes one of the major concerns. In many cases, the density of wireless sensor nodes is high, leading to high redundancy in sensor nodes' monitoring areas. In other words, a sensor node's monitoring area is usually overlapped with its nearby sensors' monitoring areas. Such redundancy

Manuscript received August 15, 2008; revised March 25, 2009 and October 29, 2009; accepted January 1, 2010. The associate editor coordinating the review of this paper and approving it for publication was B. Liang.

This work was supported in part by the National Science Foundation (NSF) under grants CCF-0829827, CNS-0716211, CNS-0737325, CNS-0721907, and CNS-0709268, as well as the Army Research Office under grants W911NF-07-1-0250 and W911NF-08-1-0334.

A. Olteanu and Y. Xiao (corresponding author) are with the Dept. of Computer Science, Univ. of Alabama, Tuscaloosa, AL 35487, USA (e-mail: aolteanu@cs.ua.edu,yangxiao@ieee.org).

$\mathrm{K}$. Wu is with the Dept. of Computer Science, Univ. of Victoria, Victoria, BC, Canada (e-mail: wkui@ieee.org).

$\mathrm{X}$. Du is with the Dept. of Computer and Information Sciences, Temple Univ., Philadelphia, PA 19122 USA (e-mail: dxj@ieee.org).

Digital Object Identifier 10.1109/TWC.2010.04.081098 is a blessing feature of wireless sensor networks for energy saving because it is unnecessary to make all sensor nodes active at the same time. Sensor nodes should be scheduled to work alternatively within certain Quality of Service (QoS) constraints in order to save energy consumption [11], [18].

One of the important QoS constraints is the chance of detecting an interesting event. Under this constraint, numerous redundancy-based sensor scheduling schemes [12], [13], [14], [18] have been proposed for energy efficiency. The state-ofthe-art mathematical models [1], [2], [3], [4], [5], [6], [7], [8], [9], [10], [11], [12], [13], [18] are targeted at the goal that each point in the field is monitored with a certain probability at any time instance. By scheduling sensor nodes to work or sleep, we are actually weaving a "net" with its grid size dynamically adjusted with the number of working sensors. Analogously, if we only want to catch fish, we should not weave a net that can actually catch shrimp. Since in real applications, the size and the shape of the monitored objects should not be ignored, it is clear that scheduling schemes based on existing point coverage models will form a network that is unnecessarily dense. Further improvement on this broadly investigated problem is possible and is of great importance for both academic research and industrial practice.

In this paper, we build a detection model that takes into consideration the impact of the size and the shape of monitored objects. More specifically, we investigate the relationship between the detection probability, the intrusion coverage intensity, the number of working sensor nodes, and the size and the shape of intrusion objects. We prove many mathematical results related to the detection probability and intrusion coverage intensity and study the asymptotic properties of these detection metrics. Our model also provides analytical results on two optimization problems: one is to find the minimum number of working sensor nodes that can detect a given object of non-negligible size with a given probability; the other is to find the maximum detection probability given the ratio of the number of sensor nodes over the size of the monitored area. We also study the problem of maximizing network lifetime under some QoS constraints. We prove the existence of the solution and derive the explicit form of the solution under certain conditions.

Our model is more accurate for applications where the shape of monitored objects (e.g., tanks) should not be modeled as points. Scheduling schemes based on our new detection model can create a working network that accurately matches the application requirement. Such a network can be useful when 
it is employed in a particular environment, as in detecting enemy tanks, or in the border, for detecting human beings crossing the border. In such cases the size of the intrusion objects, whether they are tanks or human bodies, can be easily approximated. In addition, many results in the paper remain valid for any object size. In particular, the two optimization problems in Section V are independent of the object's shape and size and are valid for any object of a given reasonable area. The problem of maximizing network lifetime and the results related to the intrusion coverage intensity provide some insights on how the network can be adapted to the size and the shape of the intruder, specifically, how many working sensors should be used and how they can be scheduled depending on the dimensions of the object we want to detect.

The rest of the paper is organized as follows. In Section II, we introduce related work. In Section III, we present a naive approach that covers a given area and detects an intrusion object of circular or rectangular shape with the minimum number of sensor nodes. In Section IV, we study a stochastic version of the problem, i.e., the probabilistic approach to detecting intrusion objects. We further investigate two optimizations related to the detection probability and the shape and the size of intrusion objects in Section V. Section VI focuses on the intrusion coverage intensity (defined later in the paper), and Section VII focuses on optimizing network lifetime. We conclude the paper in Section VIII.

\section{RELATED WORK}

Much work has focused on sensor scheduling algorithms with the purpose of achieving energy efficiency without reducing sensing coverage. One way to minimize energy consumption and extend network lifetime is to put some sensor nodes into sleep but allow others to remain active as long as the whole given area is covered. In the meantime, it is required that both coverage and network connectivity should be satisfied.

To solve the above problem, probabilistic coverage with randomized scheduling algorithms is studied. In many cases, existing research [1], [2], [3], [4], [5], [6], [7], [8], [9], [10], [11], [12], [13], [18] assumes that each point in the field should be monitored. In [11], [12], [13], [18], a special case of a randomized scheduling algorithm is investigated, where $k$ subsets of sensors work alternatively, with only one subset being active at a certain moment. Each subset contains the same number of sensors. The problem of maximizing network lifetime under Quality of Service constraints is analyzed in [11], [12], [13], [18]. The results are stated and proved in terms of the network detection probability, the detection delay, and the intrusion coverage intensity. The detection probability is the probability that an intrusion event is detected, e.g., an enemy object enters the monitored area. The detection delay is defined as the average delay with respect to the scheduling rounds to detect such an event. The intrusion coverage intensity is defined as the probability that a given area is detected at any given time by at least one active sensor. For more results concerning energy-efficient joint estimation in sensor networks, please refer to [11], [12], [13], [18].

Xiao et al. [14], [19] study another case of the randomized scheduling algorithm to detect intrusion objects with a large

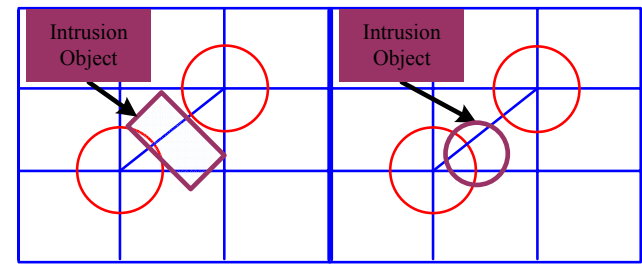

Fig. 1. Sensors are placed at the intersections of a virtual grid. The circles represent the areas covered by sensors and the intrusion object is approximated as a rectangle (left) or disk (right).

size, in which case treating the objects as points may be too conservative. Using the same model, in this paper we perform a comprehensive study on the detection of an intrusion object that has a non-negligible size (such as a tank). Along the line, the relevant optimization problems can be solved as well. This is the purpose of the present paper.

\section{A NAIVE APPROACH}

In this section, we describe a simple approach to answering the following question: how to use the minimum number of sensor nodes to cover a given rectangular area such that an intrusion object with a non-negligible size can be detected?

To simplify analysis, we assume that a sensor node's sensing coverage can be approximated as a circular area. Assume that the whole rectangular area is divided by a virtual grid. Also assume that the sensor nodes are placed at the intersections of the virtual grid. We first determine the maximum size of the square cells in the virtual grid, with which we can then easily estimate the minimum number of nodes needed to cover the area.

Let $d$ denote the diameter of the square cells in the virtual grid. Let $o$ denote the size of the intrusion object. We use two typical examples to illustrate our calculation.

- Case 1: Assume that the intrusion object can be approximated as a rectangular shape, with side length of $b$ and $\frac{o}{b}$, respectively. Let $r$ be the size of the circular area monitored by each sensor. It is easy to see that the radius of the circular area is $\sqrt{\frac{r}{\pi}}$. We can then choose to divide the whole area into squares such that the diameter $d$ of each square satisfies $d<\min \left\{b, \frac{o}{b}\right\}+2 \sqrt{\frac{r}{\pi}}$, as shown in the left of Fig. 1.

- Case 2: Assume that the intrusion object can be approximated as a disk. We can choose $d<2 \sqrt{\frac{o}{\pi}}+2 \sqrt{\frac{r}{\pi}}$, as shown in the right of Fig. 1.

By choosing the diameters the above way, we ensure that no matter how we place an intrusion object on the grid, its boundary will overlap with at least one sensor's monitoring area, i.e., the object cannot escape the detection.

Having a constraint on the diameter of the square cells, we can easily derive an upper bound on the edge length of the squares, $l_{\text {square }}$ :

$$
l_{\text {square }}< \begin{cases}\left(\min \left\{b, \frac{o}{b}\right\}+2 \sqrt{\frac{T}{\pi}}\right) / \sqrt{2}, & \text { Case } 1 ; \\ \left(2 \sqrt{\frac{o}{\pi}}+2 \sqrt{\frac{r}{\pi}}\right) / \sqrt{2}, & \text { Case } 2 .\end{cases}
$$

While this method of placing sensor nodes can provide deterministic intrusion detection, it requires that all sensor nodes to be active at any given time, which may deplete 


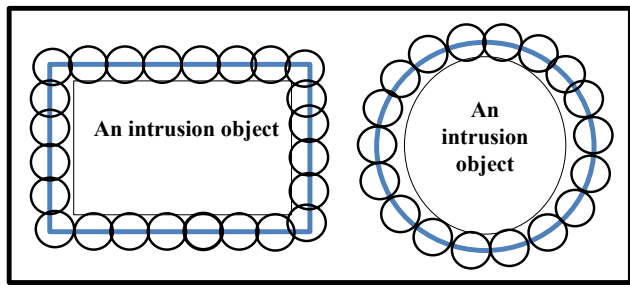

Fig. 2. The sensing areas intersect with the surface of an intrusion object.

sensors' energy quickly. In addition, placing sensor nodes accurately on the intersections of a grid may not be an easy job and may require high human labor. As such, we investigate the probabilistic methods in the following sections.

\section{Probabilistic Methods for Detecting INTRUSION}

Assume that $n$ sensors are randomly deployed in a field. To simplify analysis, we assume that the field is much larger than a sensor's sensing range and as such the boundary effort can be ignored. We assume that the $n$ sensors are evenly put into $k$ disjoint subsets and the $k$ subsets work according to the round-robin scheduling. We also assume that the network is homogeneous and the intrusion object is completely contained in the network. Therefore our results are independent of the location of the object. We are interested in the intrusion coverage intensity and the detection probability, which are defined later.

Let $r$ be the size of the sensing area of each sensor and $a$ the size of the whole sensing field. The probability that each sensor covers a given point in the field is $r / a$. Since every sensor is scheduled in one of the $k$ subsets, and there are $n / k$ sensors in each subset, then $[1-r / a]^{n / k}$ represents the probability that a given point is not covered by any of the active sensors. Following this line of reasoning, the probability that a point in the field is detected by at least one sensor at any given time is $1-[1-r / a]^{n / k}$.

In the following, we derive the probability that a sensor's sensing area intersects the surface of an intrusion object, denoted as $p_{1}$. As shown in Fig. 2, any sensor within the boundary of the grey lines can detect the intrusion objects. Let the area bounded by the grey lines shown in Fig. 2 (left and right) be denoted by $o_{1}$.

- Case 1: Assume that the intrusion object can be approximated as a disk of size $o$. It is easy to see that the size of the area $o_{1}$ bounded by the grey lines as shown in Fig. 2 (right) could be calculated by

$$
\pi\left(\sqrt{\frac{o}{\pi}}+\sqrt{\frac{r}{\pi}}\right)^{2}=(\sqrt{r}+\sqrt{o})^{2} .
$$

In this case,

$$
p_{1}=(\sqrt{r}+\sqrt{o})^{2} / a .
$$

- Case 2: Assume that the intrusion object can be approximated as a rectangular shape with side length of $b$ and $o / b$, respectively. The size of the area $o_{1}$ as shown in Fig. 2(left) could be calculated by

$$
o+2 b \sqrt{\frac{r}{\pi}}+2 \frac{o}{b} \sqrt{\frac{r}{\pi}}+4 r \frac{1}{4}=o+2\left(b+\frac{o}{b}\right) \sqrt{\frac{r}{\pi}}+r,
$$

which is obtained by adding the areas of 4 smaller rectangles: two with side length of $b$ and $\sqrt{\frac{r}{\pi}}$, and two with side length $\frac{o}{b}$ and $\sqrt{\frac{r}{\pi}}$, plus 4 quarters of a disk of area $r$ (at the corners), to the initial object area $o$. In this case,

$$
p_{1}=\left(o+2\left(b+\frac{o}{b}\right) \sqrt{\frac{r}{\pi}}+r\right) / a
$$

With $p_{1}$ calculated, we can obtain the intrusion coverage intensity, which is defined as the probability that any intrusion object is detected by at least one sensor at any given time. Since there are $n$ sensors and $k$ rounds in total, the probability that the intrusion object is not detected by any active sensor, at any time, is $\left[1-p_{1}\right]^{n / k}$. Then the intrusion coverage intensity is given by $V_{n}=1-\left[1-p_{1}\right]^{n / k}$.

The intrusion coverage intensity discloses how well the whole area is covered with respect to the detection of intrusion objects with non-negligible size. Assume that $L$ is the duration of an intrusion event and $T$ is the length of a round-robin scheduling round. We need to know the probability that the intrusion event is detected by at least one sensor. This probability is called detection probability and is denoted as $P_{d}$. By replacing $r / a$ with $p_{1}$ in [11], [12], [13], [18] ${ }^{1}$, we obtain:

$$
P_{d}=\left\{\begin{array}{l}
1-\left(1-p_{1}\right)^{n}, \quad L \geq(k-1) T \\
1-(1-s)\left(1-p_{1} \frac{\lceil L / T\rceil}{k}\right)^{n} \\
\quad-s\left(1-p_{1} \frac{\lceil L / T\rceil+1}{k}\right)^{n}, L<(k-1) T
\end{array}\right.
$$

This expression depends on $\lceil L / T\rceil, n, k, r / a$, and $s$, where $s=(L / T+1-\lceil L / T\rceil)$. The assumption is that $L$ may not be a natural multiple of $T$.

\section{The Properties of Detection Probability}

\section{A. Constrained Optimal Detection Probability}

Having derived the analytical expression of the detection probability $P_{d}$, we now investigate the constrained optimal intrusion detection by solving two main optimization problems and their variations. The results provided by these two optimization problems are independent of the object's shape and size and are valid for any object of a given area $o$, reasonably assuming that $o$ is smaller than the total area to monitor. We also determine a lower bound and an upper bound on the detection probability.

The first problem is concerned with maximizing/minimizing the detection probability $P_{d}$ under different constraints. For instance, we may put the constraint on the ratio of $n / a$ (i.e., the ratio of the number of sensors over the size of the whole area), since this ratio represents the density of the sensor nodes and thus the system cost. For comparison, we use $1 / r$ as a measure of benchmark, because this measure means the ideal situation: one sensor per area of size $r$.

Optimization Problem 1: Determine the sufficient conditions for finding the maximum value and the minimum value

\footnotetext{
${ }^{1}$ Note that there is a slight difference in the model used in [11], [12], [13], [18], in which each sensor randomly joins one of the $k$ subsets. Nevertheless, the derivation of $P_{d}$ in [11], [12], [13], [18] holds true for the model in this paper.
} 
of $P_{d}$ under the constraint that $n / a \leq u_{0}$, where $u_{0}$ is a given positive constant.

By changing the value of $u_{0}$, particularly by checking whether or not $u_{0}$ is larger than $1 / r$, we obtain the properties of detection probability in different scenarios. To ease derivation, we will use $x$, a real number, instead of $n$, to make the differential calculation possible. In the above optimization problem, since the detection probability $P_{d}$ is considered as a function of two variables, the number of sensors $x$ and the size of the whole area $a$, we rewrite $P_{d}$ as $P_{d}(x, a)$ and build a Cartesian coordinate system with the $\mathrm{x}$-coordinate representing the number of sensors and the y-coordinate representing the size of the whole area. To obtain results meaningful in practice, we use a rectangle $R_{1}=\left[4, u_{0} a_{0}\right] \times\left[4 r, a_{0}\right]$ on the Cartesian coordinate system to confine the variables, i.e., $4 \leq x \leq u_{0} a_{0}$ and $4 r \leq a \leq a_{0}$. This limitation is to exclude certain uninteresting scenarios, e.g., the number of sensors is too small (smaller than 4), or the size of the whole area is too small (smaller than $4 r$ ). We put an upper bound $a_{0}$ on the size of the whole area, because it is impractical to consider an area having infinite size.

The following results hold for intrusion objects with either circular or rectangular shape.

Theorem 1 provides the maximum and minimum values of $P_{d}(x, a)$ on rectangle $R_{1}$ under constraint $x / a \leq u_{0}$ where $u_{0} \geq 1 / r$. It also provides the maximum and minimum values of $P_{d}(x, a)$ with constraint $x=a u_{0}$ on the same rectangle.

Theorem 1: Consider the problem of finding $\max _{R_{1}} P_{d}(x, a)$ and $\min _{R_{1}} P_{d}(x, a)$ with the variables confined in $R_{1}=$ $\left[4, u_{0} a_{0}\right] \times\left[4 r, a_{0}\right]$ and with the constraint $x / a<u_{0}$ where $u_{0}$ is a given positive constant greater than $1 / r$. We have $\max _{R_{1}} P_{d}(x, a)=P_{d}\left(4 r u_{0}, 4 r\right)$ and $\min _{R_{1}} P_{d}(x, a)=$ $P_{d}\left(4, a_{0}\right)$. If we change the constraint $x / a<u_{0}$ to $x / a=u_{0}$, we have $\max _{R_{1}} P_{d}(x, a)=P_{d}\left(4 r u_{0}, 4 r\right)$ and $\min _{R_{1}} P_{d}(x, a)=$ $P_{d}\left(u_{0} a_{0}, a_{0}\right)$.

As shown in Fig. 3a, the trapeze $T=T_{M N P Q}$ has vertices $M\left(4 r u_{0}, 4 r\right), N\left(u_{0} a_{0}, a_{0}\right), P\left(4, a_{0}\right)$ and $Q(4,4 r)$. Theorem 1 indicates that when $x / a<u_{0}$, the maximum value and the minimum value of $P_{d}(x, a)$ is reached at vertex $M$ and vertex $P$, respectively. When $x / a=u_{0}$, this constraint defines a line segment with end points $M$ and $N$, and $P_{d}(x, a)$ reaches the maximum value and the minimum value at points $M$ and $N$, respectively.

The following corollary considers the special case $u_{0}=$ $1 / r$, that is, the trapeze becomes triangle $T_{M N P}$, and the vertices $M, N$ and $P$ are given by $(4,4 r),\left(a_{0} / r, a_{0}\right)$ and $\left(4, a_{0}\right)$, respectively, as shown in Fig. $3 \mathrm{~b}$.

Corollary 1: Let the optimization problem be as in Theorem 1. If $x / a<u_{0}$ and $u_{0}=1 / r$, then $\max _{R_{1}} P_{d}(x, a)=$ $P_{d}(4,4 r)$ and $\min _{R_{1}} P_{d}(x, a)=P_{d}\left(4, a_{0}\right)$. If $x / a=u_{0}$ and $u_{0}=1 / r$, then $\max _{R_{1}} P_{d}=P_{d}(4,4 r)$ and $\min _{R_{1}} P_{d}=$ $P_{d}\left(a_{0} / r, a_{0}\right)$.

Theorem 1 and Corollary 1 present the results when $u_{0}>$ $1 / r$ and $u_{0}=1 / r$, respectively. Theorem 2 considers the case where $u_{0}<1 / r$.

Theorem 2: Consider the problem of finding $\max P_{d}(x, a)$ and $\min _{R_{1}} P_{d}(x, a)$ with the variables confined in $R_{1}=$ $\left[4, u_{0} a_{0}\right] \times\left[4 r, a_{0}\right]$ and with the constraint $x / a<u_{0}$ where $u_{0}$ is a given positive constant smaller than $1 / r$. We have $\max _{R_{1}} P_{d}(x, a)=P_{d}\left(4,4 / u_{0}\right)$ and $\min _{R_{1}} P_{d}(x, a)=P_{d}\left(4, a_{0}\right)$. If we change the constraint $x / a<u_{0}$ to $x / a=u_{0}$, we have $\max _{R_{1}} P_{d}(x, a)=P_{d}\left(4,4 / u_{0}\right)$ and $\min _{R_{1}} P_{d}(x, a)=$ $P_{d}\left(u_{0} a_{0}, a_{0}\right)$.

The above constraints determine a triangle $T_{M N P}$ inside rectangle $R_{1}$, shown in Fig. 3c. The vertices $M, N$ and $P$ of the triangle have coordinates: $\left(4,4 / u_{0}\right),\left(a_{0} u_{0}, a_{0}\right)$ and $\left(4, a_{0}\right)$, respectively. Theorem 2 indicates that when $x / a<u_{0}$, the maximum value and the minimum value of $P_{d}(x, a)$ is reached at vertex $M$ and vertex $P$, respectively. When $x / a=u_{0}$, the constraints define a line segment with end points $M$ and $N$, and $P_{d}(x, a)$ reaches the maximum value and the minimum value at points $M$ and $N$, respectively.

It is interesting to see the practical meaning that Theorems 1 and 2 deliver. In both cases, the minimum detection probability is the case when the minimum number of sensors is used to cover the entire area, as we would have expected intuitively. If we look at the maximum detection probability, in the case of Theorem 1, maximum detection is achieved when the area is minimal and the number of sensors is an intermediary value between 4 and $a_{0} u_{0}$, while in the case of Theorem 2 , maximum detection is reached when the number of sensors equals 4 and the size of the area equals $4 / u_{0}$.

The second problem we consider consists in the reverse optimization problem: minimize the ratio of the number of sensors over the size of the whole area, given a certain detection probability or an upper bound on the detection probability.

Optimization Problem 2: Find $\min (x / a)$, with constraint $P_{d}=P_{0}$ where $P_{0}$ is a constant greater than zero. If possible, find tighter lower and upper bounds on $P_{0}\left(0<P_{0}<1\right)$.

The restriction $P_{d}=P_{0}$ defines implicitly $x$ as a function of $a$ and conversely $a$ as a function of $x$, if we do not assume that $a$ is given a prior. We thus use the implicit function theorem [17] to solve the problem. As before, we confine the variables, the number of sensors $x$ and the area to be covered $a$, within a rectangle $R_{2}:=\left[4, n_{0}\right] \times\left[4 r, a_{0}\right]$, where $n_{0}$ is a natural number and the upper bound on the number of sensors. Let $k_{0}:=n_{0} / m$, where $m$ is the number of sensors in each of the $k$ subsets. To obtain results, we need to assure that the curve $P_{d}=P_{0}$ intersects the interior of rectangle $R_{2}$. The condition $L>T\left(k_{0}-1\right)$ allows us to express $x$ explicitly as a function of $a$. Here, again, we have an optimization problem of two variables: the number of sensors $x$ and the area to be covered, $a$. However, by expressing $x$ as a function of $a$, we are able to convert the problem to an optimization problem in only one variable $a$.

Theorem 3: Assume that $P_{d}\left(4, a_{0}\right)<P_{0}<P_{d}\left(n_{0}, a_{0}\right)$ and that $P_{d}\left(4, a_{0}\right)<P_{0}<P_{d}(4,4 r)$. Also assume that for any $a \in\left[a(4), a_{0}\right]$ there exists $x=x(a) \in\left[4, x\left(a_{0}\right)\right]$ such that $P_{d}(x(a), a)=P_{0}$.

1) Then there exist strictly increasing functions,

$$
x=x(a), a=a(x),
$$




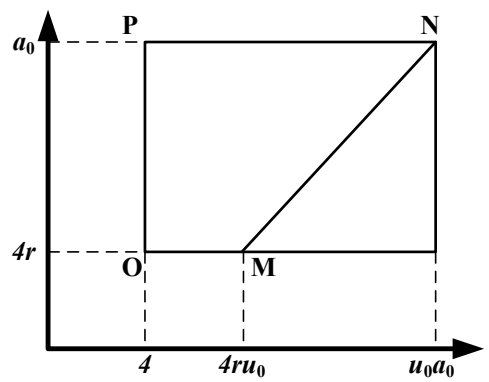

(a)

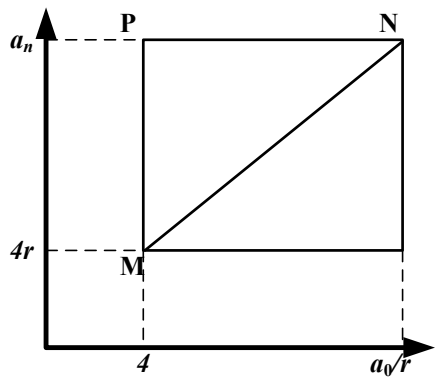

(b)

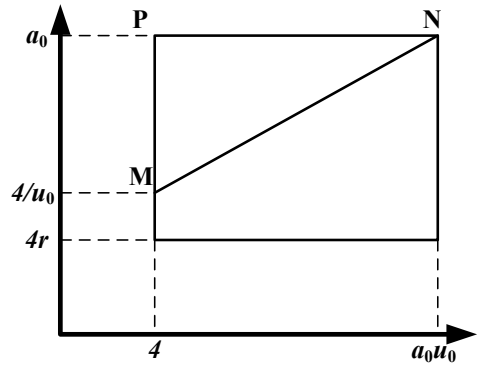

(c)

Fig. 3. (a) Constraints $x / a \leq u_{0}$ and $u_{0} \geq 1 / r$ determine trapeze MNPQ inside rectangle $R_{1}=\left[4, u_{0} a_{0}\right] \times\left[4 r, a_{0}\right]$. (b) Constraint $x / a \leq u_{0}=1 / r$ determines triangle MNP inside rectangle $\left[4, a_{0} / r\right] \times\left[4 r, a_{0}\right]$. (c) Constraints $x / a \leq u_{0}$ and $u_{0}<1 / r$ determine triangle MNP inside rectangle $R_{1}=$ $\left[4, u_{0} a_{0}\right] \times\left[4 r, a_{0}\right]$.

inverses of each other, such that $P_{d}(x, a)=P_{0}$. Function $\frac{x(a)}{a}$ is increasing with $a$, hence

$$
\min \left\{\frac{x(a)}{a} \mid P_{d}(x(a), a)=P_{0}, a \in\left[a(4), a_{0}\right]\right\}=\frac{4}{a(4)} .
$$

2) If in addition we assume that $L \geq(k-1) T$, we have: $x(a)=\frac{\ln \left(1-P_{0}\right)}{\ln \left(1-o_{1} / a\right)}, a(x)=\frac{o_{1}}{1-\left(1-P_{0}\right)^{1 / x}}$, which leads to

$\min \left\{\frac{x(a)}{a} \mid P_{d}(x(a), a)=P_{0}\right\}=\frac{4}{o_{1}}\left[1-\left(1-P_{0}\right)^{1 / 4}\right]$.

The minimum ratio of the number of sensors over the size of the whole area, given a desired detection probability, can be reached when the minimum number of sensors is used for area size $a(4)$. We have also deduced a constrained minimum ratio.

The next result represents the upper and lower bounds for $P_{d}$.

Theorem 4: The following inequalities stand:

$$
1-\left[1-\frac{\left\lceil\frac{L}{T}\right\rceil}{k} p_{1}\right]^{n}<P_{d}<1-\left[1-\frac{\left\lceil\frac{L}{T}\right\rceil+1}{k} p_{1}\right]^{n} .
$$

Theorem 4 presents some very simple, tight bounds for $P_{d}$. We can see that $s$ has been eliminated from the rather complicated expression of $P_{d}$ in (3). Instead of using formula (3) we can now use either one of the two bounds in Theorem 4 as an approximation of $P_{d}$.

\section{B. Detection Probability Related To The Shape Of The Intru- sion Object}

Intuitively, the detection probability increases as the size of the intrusion object increases. But it is unclear for two rectangular intrusion objects, which one is easier to be detected: a square object or a long thin strip? We answer this question in this subsection.

Assume that the size of the rectangular intrusion object is $o$, and its one side length is $b$. In the following we study $P_{d}$ as a function of $b$, shifting the problem from the size to the shape of the intrusion object. Lemma 1 determines the signature of the derivative of $p_{1}$ as a function of $b$, and Lemma 2 determines the signature of the derivative of $P_{d}$ as a function of $b$. We show that the minimum detection probability is reached when $b=\sqrt{o}$, i.e., when the intrusion object is a square. We then give an asymptotic result for $P_{d} \min$.

Lemma 1: The signature of $\frac{d p_{1}}{d b}$ is:

$$
\frac{d p_{1}}{d b}(b)\left\{\begin{array}{l}
<0, b<\sqrt{o}, \\
\geq 0, b \geq \sqrt{o},
\end{array}\right.
$$

Therefore, $p_{1}$ decreases on $[0, \sqrt{o}]$, and increases on the right of $\sqrt{o}$.

Lemma 2: $\operatorname{sign}\left(\frac{d P_{d}}{d b}(b)\right)=\operatorname{sign}\left(\frac{d p_{1}}{d b}\right)$.

Lemma 2 shows that $P_{d}$ behaves similarly to $p_{1}$, so $\sqrt{o}$ is also a minimum point for $P_{d}$. Therefore a square shaped intrusion object is most likely to go undetected.

By replacing $b$ with $\sqrt{o}$ in the expression of $p_{1}$ for a rectangle (see Section IV), we obtain the following expression of $P_{d \min }$ :

$1-(1-s)\left[1-\frac{\left\lceil\frac{L}{T}\right\rceil}{k} p_{1 \min }\right]^{n}-s\left[1-\frac{\left\lceil\frac{L}{T}\right\rceil+1}{k} p_{1 \min }\right]^{n}$,

where $p_{1 \min }=\frac{1}{a}\left[o+4 \sqrt{o} \sqrt{\frac{r}{\pi}}+r\right]$. If $m$ is the number of sensors per subset (i.e., $k=n / m$ ), we have the following asymptotic result:

$$
\lim _{n \rightarrow \infty} P_{d \min }=1-(1-s) e^{-m\left\lceil\frac{L}{T}\right\rceil p_{1} \min }-s e^{-m\left(\left\lceil\frac{L}{T}\right\rceil+1\right) p_{1} \min } .
$$

\section{The Properties of Intrusion Coverage INTENSITY}

We denote the intrusion coverage intensity as $V(x, o)$ if we treat the intrusion coverage intensity as a function of two variables, the number of sensors $x$ and the size of the intrusion object $o$. From Section IV, by replacing $p_{1}$ as a function of $o$, we have the following expressions:

$$
V(x, o)=1-\left[1-\frac{1}{a}(\sqrt{r}+\sqrt{o})^{2}\right]^{x / k}
$$

for a circular intrusion object, and

$$
V(x, o)=1-\left[1-\frac{1}{a}(o+r+2 \sqrt{r / \pi}(b+o / . b))\right]^{x / k}
$$

for a rectangular intrusion object, respectively.

To study the properties of $V(x, o)$, we build a Cartesian coordinate system with the $x$-coordinate representing the number of sensors and the $y$-coordinate representing the size of a sensor's coverage area. Similar as before, we confine the variables in an area, denoted as $S$, over the Cartesian 


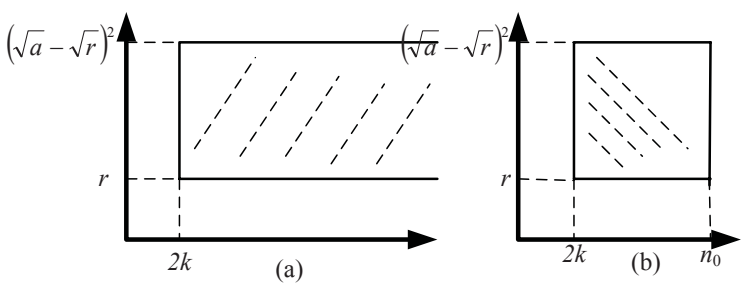

Fig. 4. (a) Strip $S=[2 k, \infty) \times\left[r,(\sqrt{a}-\sqrt{r})^{2}\right]$. (b) Rectangle $R_{3}$ included in strip $S=[2 k, \infty) \times\left[r,(\sqrt{a}-\sqrt{r})^{2}\right]$.

coordinate system. Theorem 5 finds a point over $S$ that minimizes $V(x, o)$ when the intrusion object has a disk shape.

Theorem 5: If we define an area over the Cartesian coordinate system, $S=[2 k, \infty) \times\left[r,(\sqrt{a}-\sqrt{r})^{2}\right],(\sqrt{a}-\sqrt{r})^{2}>$ $o$ and $o>r$, then $\min _{S} V(x, o)=V(2 k, r)=1-(1-4 r / a)^{2}$.

The reason that we confine the variables within $S$ as shown in Fig. 4a is as follows. When the intrusion object has a disk shape, we have $(\sqrt{r}+\sqrt{o})^{2} / a=p_{1} \leq 1$. This leads to $o \leq$ $(\sqrt{a}-\sqrt{r})^{2}$. In addition, it is only interesting to assume $o \geq r$ because we only want to detect objects with a large size. We also assume that the number of sensors is large enough, e.g., larger than $2 k$.

The following corollary takes the supermum over all points in the set $S$ and shows that it is upper-bounded by a finite number.

\section{Corollary 2:}

$$
\begin{aligned}
& \sup \left(V(x, o)-\frac{\partial V}{\partial x}(2 k, r)(x-2 k)-\frac{\partial V}{\partial o}(2 k, r)(o-r)\right) \\
& \leq V(2 k, r) \\
& =1-(1-4 r / a) \\
& =1-e^{2 \ln (1-4 r / a)} \\
& \leq 1-e^{2 \frac{-4 r / a}{1-4 / a}} \\
& =1-e^{-\frac{8 r}{a-4 r}} .
\end{aligned}
$$

If we put an upper bound on the number of sensors, i.e., $x \leq n_{0}$, we reduce the area $S$ to a rectangle $R_{3}=$ $\left[2 k, n_{0}\right] \times\left[r,(\sqrt{a}-\sqrt{r})^{2}\right]$ over the Cartesian coordinate system, as shown in Fig. 4b. The following theorem shows that with variables confined in $R_{3}$, the maximum and minimum intrusion coverage intensity are reached.

Theorem 6: $\max _{(x, o) \in R_{3}} V(x, o)=V\left(n_{0},(\sqrt{a}-\sqrt{r})^{2}\right)=1$ and $\min _{(x, o) \in R_{3}} V(x, o)=V(2 k, r)=1-\left(1-\frac{4 r}{a}\right)^{2}$.

Based on the previous result, we obtain the following bounds.

Corollary 3: $1-e^{-\frac{8 r}{a}}<1-e^{2 \ln \left(1-\frac{4 r}{a}\right)} \leq V(x, o) \leq 1$, for all $(x, o) \in R_{3}$.

Corollary 4: Let $2 k<x<n_{0}$ and let $k=\frac{n_{0}}{m}$. The following asymptotic result stands: $1-e^{-\frac{8 r}{a}} \leq V(x, o) \leq 1-e^{-m}$.

In the following, we consider $V$ as a function of $x$ and $b$ and we establish the upper and lower bounds for $V(x, b)$.

Theorem 7: Let $o$ be a fixed constant, $o=b \frac{o}{b}$, where $b$ is variable. Assume that $x \in\left[4, n_{0}\right]$ and $\sqrt{o} \in\left[b_{1}, b_{2}\right]$. We have

$$
\begin{aligned}
& 1-\left[1-\frac{1}{a}\left(o+r+4 \sqrt{\frac{r}{\pi}} \sqrt{o}\right)\right]^{4 / k} \\
\leq & V(x, b) \\
\leq & \max _{j \in\{1,2\}}\left\{1-\left(1-\frac{o+r+\sqrt{r / \pi}\left(b_{j}+o / b_{j}\right)}{a}\right)^{n_{0} / k}\right\} .
\end{aligned}
$$

The last result of this section uses some well-known inequalities to obtain the upper and lower bounds for $V$ only as a function of the number of sensors, $x$.

Theorem 8: $\max \left\{1-e^{-p_{1} m}, \frac{3-4 e^{-p_{1} m}}{4\left(1-e^{-p_{1} m}\right)}\right\} \leq V_{n} \leq 1-$ $e^{-\frac{m p_{1}}{1-p_{1}}}$

We summarize the analytical results of this section and their practical meaning as follows.

- When the intrusion object has a disk shape, we have found the minimum intrusion coverage intensity on an unbounded strip (Theorem 5) and have shown that this value can be upper bounded by a finite number (Corollary 2). By putting a limit on the number of sensors, we further found the minimum and maximum coverage intensities (Theorem 6).

- When the intrusion object has a rectangular shape, the intrusion coverage intensity becomes a function of the number of nodes and the side length of the rectangle. We have found upper and lower bounds for the coverage intensity (Theorem 7). By fixing the size of the object, the intrusion coverage intensity is only a function of the number of sensor nodes and its upper and lower bounds can be calculated as well (Theorem 8).

- Corollary 3 and Theorem 8 imply that if the intrusion object is large, then the ratio $n / k$ (the total number of sensors over the number of subsets) should be kept small. Likewise, if the intrusion object is small, the ratio $n / k$ could be large. These results provide some insights on the relationship between the size of the object and the active sensors required in each round of scheduling.

\section{Optimizing Network Lifetime}

Maximizing the network lifetime leads in fact to maximizing the number subsets of sensors $(k)$ that work alternatively [11], [12], [13], [18]. Following the idea in [11], [12], [13], [18], we look to maximize $k$ as a function of the size of the intrusion objects, $o$, with the following constraints: 1) $P_{d}=P_{0}$, 2) fixed number of sensors $\left.n, 3\right) \operatorname{QoS}_{V_{n}}$, and 4) bounds on the object size, where $P_{0}$ is a given constant between 0 and 1 and $\operatorname{QoS}_{V_{n}}$ is a predefined Quality of Service (QoS) constraint. We are able to obtain the form of the solution, not only its existence as in [11], [12], [13], [18]. In addition, by choosing equality constraints for the detection probability, we derive the explicit expression of function $k(o)$. Theorem 9 is for an intrusion object shaped as a disk, while Theorem 10 refers to an intrusion object shaped as a rectangle. In the following, let $Q=\lceil L / T\rceil$.

Theorem 9: Consider an intrusion object that has a disk shape. Let $o_{1}>0$ and let be a given natural number such that: a) $\left(\sqrt{o_{1}}+\sqrt{r}\right)^{2} \geq a\left[1-\left(1-Q_{o} S_{V_{n}}\right)^{1 / c}\right]$; 
b) $o_{1} \leq(\sqrt{a}-\sqrt{r})^{2} ; \quad$ c) There exists $P_{0}$ such that $P_{0}=1-(1-s)\left[1-\frac{Q}{a}\left(\sqrt{r}+\sqrt{o_{1}}\right)^{2}\right]^{c}-$ $s\left[1-\frac{Q+1}{a}\left(\sqrt{r}+\sqrt{o_{1}}\right)^{2}\right]^{c} ;$ d) There exists $k_{1}=$ $k_{1}\left((\sqrt{a}-\sqrt{r})^{2}\right)$ such that $P_{d}\left((\sqrt{a}-\sqrt{r})^{2}, k_{1}\right)=P_{0}$; in addition $k_{1} \leq \frac{1}{1-\left(1-Q \circ S_{V_{n}}\right)^{1 / n}}$. Then the problem of maximizing $k$ with constraints: i) $P_{d}=P_{0}$; ii) $1 \leq k \leq \frac{(\sqrt{r}+\sqrt{o})^{2}}{a\left[1-\left(1-Q o S_{V_{n}}\right)^{1 / n}\right]}$; iii) $n=c$, where $c$ is a given constant; iv) $o_{1} \leq o \leq(\sqrt{a}-\sqrt{r})^{2}$; has the solution $\max k=\left[a /\left(\sqrt{o_{1}}+\sqrt{r}\right)^{2}\right\rfloor$. Furthermore, from constraint (i.) we obtain an explicit expression for $k$ : $k(o)=\left(\frac{\sqrt{r}+\sqrt{o}}{\sqrt{r}+\sqrt{o_{1}}}\right)^{2}$.

Note that Constraint ii. is derived from the QoS constraint $V_{n} \geq Q o S_{V_{n}}>0$, which can be rewritten as $1 \leq k \leq$ $\frac{(\sqrt{r}+\sqrt{o})^{2}}{a\left[1-\left(1-Q o S_{V_{n}}\right)^{1 / n}\right]}$ using the definition of $V_{n}$. Constraint iii. shows that we keep $n$ fixed and we only modify $k$. Constraint iv. is to assume that $o$ is larger than some number $o_{1}$. Constraint iv. is motivated by the fact that the circular object plus its shadow (see Fig. 2 and Section IV), given by $(\sqrt{r}+\sqrt{o})^{2}$, should be smaller than $a$, the area of the whole region. Conditions a.-d. are related to the Constraints i.-iv., and are used to obtain the solution faster.

Theorem 10: Consider an intrusion object that has a rectangular shape. Let $o$ be the area of the intrusion object, $b_{1}, b_{2}>0$ such that $b_{1}+o / b_{1} \leq b_{2}+o / b_{2}$, let $c$ be a natural number and $P_{0} \in[0,1]$ such that: a) $o+r+4 \sqrt{r / \pi} \sqrt{o} \geq a\left[1-\left(1-Q_{o} S_{V_{n}}\right)^{1 / c}\right] ;$ b) $b_{1}<$ $\sqrt{o}<b_{2}$, where $b_{2}=b_{\max }$ satisfies the equation $o+$ $2 \sqrt{r / \pi}\left(b_{2}+o / b_{2}\right)+r=a$; c) There exists $P_{0}$ such that $P_{0}=1-(1-s)\left[1-\frac{Q}{a}\left(r+o+2 \sqrt{r / \pi}\left(b_{1}+o / b_{1}\right)\right)\right]^{c}-$ $s\left[1-\frac{Q+1}{a}\left(r+o+2 \sqrt{r / \pi}\left(b_{1}+o / b_{1}\right)\right)\right]^{c} ;$ d) There exists $k_{2}=k_{2}\left(b_{2}\right)$ such that $P_{d}\left(b_{2}, k_{2}\right)=P_{0}, k_{2} \leq$ $\frac{1}{1-\left(1-Q o S_{V_{n}}\right)^{1 / c}}$. Then the problem of maximizing $k$ with constraints: i) $P_{d}=P_{0}$; ii) $1 \leq k \leq \frac{o+2 \sqrt{r / \pi}(b+o / b)+r}{a\left[1-\left(1-Q o S_{V_{n}}\right)^{1 / n}\right]}$; iii) $n=c$, where $c$ is a given constant; iv) $b_{1} \leq b \leq b_{2}$; has the solution $\max k=\left\lfloor a /\left(o+2 \sqrt{r / \pi}\left(b_{1}+o / b_{1}\right)+r\right)\right]$. Furthermore, from constraint (i.) we obtain an explicit expression for $k: k(b)=\frac{o+2 \sqrt{r / \pi}(b+o / b)+r}{o+2 \sqrt{r / \pi}\left(b_{1}+o / b_{1}\right)+r}$.

The practical meaning of Theorems 9 and 10 is that the number of sensor subsets $k$ can be selected and tuned based on the size and the shape of the intrusion object. These results provide some guidelines on how the network should be adapted to the size and the shape of an intruder.

\section{CONCLUSIONS}

In real applications with wireless sensor networks, the size and the shape of objects are usually non-negligible. Traditional coverage models mainly focus on monitoring each point in the field with a high probability. As a result, scheduling decisions based on existing coverage models create sensor networks that are unnecessarily dense. In this paper, we remedy this problem by proposing a more accurate coverage model that discloses the relationship among detection probability, coverage intensity, and the size and the shape of intrusion objects. We investigate and solve several important optimization problems in this model. Our analytical results provide researchers and practitioners with insights on how to build an effective sensor network to detect large objects with a small number of active sensors.

\section{APPENDIX}

Proof: (Theorem 1) To prove that the minimum detection probability is reached at point $\left(4, a_{0}\right)$, we compute the difference between the value of $P_{d}$ in a random point $(x, a)$, inside trapeze $T$, and $P_{d}\left(4, a_{0}\right)$, and show that this difference is always positive. For this we first compute the partial derivatives of $P_{d}$ with respect to both $x$ and $a$ and establish their sign.

$$
\begin{aligned}
& \frac{\partial P_{d}}{\partial x}(x, a)= \\
& -(1-s)\left[1-\frac{Q}{a k}(\sqrt{o}+\sqrt{r})^{2}\right]^{x} \ln \left(1-\frac{Q}{a k}(\sqrt{o}+\sqrt{r})^{2}\right) \\
& \quad-s\left[1-\frac{Q+1}{a k}(\sqrt{o}+\sqrt{r})^{2}\right]^{x} \ln \left(1-\frac{Q+1}{a k}(\sqrt{o}+\sqrt{r})^{2}\right) \\
& >0 . \\
& \frac{\partial P_{d}}{\partial a}(x, a)= \\
& -x\left[(1-s)\left(1-\frac{Q}{a k}(\sqrt{r}+\sqrt{o})^{2}\right)^{x-1} \frac{Q}{a^{2} k}(\sqrt{r}+\sqrt{o})^{2}\right. \\
& \left.\quad+s\left(1-\frac{Q+1}{a k}(\sqrt{o}+\sqrt{r})^{2}\right)^{x-1} \frac{Q+1}{a^{2} k}(\sqrt{r}+\sqrt{o})^{2}\right] \\
& <0 . \quad
\end{aligned}
$$

Next, using Lagrange's mean value theorem [15], let us consider the difference:

$$
\begin{aligned}
& P_{d}(x, a)-P_{d}\left(4, a_{0}\right) \\
& =\frac{\partial P_{d}}{\partial x}\left(\rho_{1}, \rho_{2}\right)(x-4)+\frac{\partial P_{d}}{\partial a}\left(\rho_{1}, \rho_{2}\right)\left(a-a_{0}\right) \\
& \geq 0
\end{aligned}
$$

Therefore, $P_{d}(x, a) \geq P_{d}\left(4, a_{0}\right)$ for any $(x, a) \in T$.

Hence, minimum $P_{d}$ is reached at $\left(4, a_{0}\right)$.

A more complicated solution is needed for the maximization problem. Since we want to show that at $\left(4 r u_{0}, 4 r\right) P_{d}$ reaches the maximum value, the previous technique cannot be used as we do not know the sign of $x-4 r u_{0}$. Therefore, to find the maximum of $P_{d}$ we first use the fact that, according to Fermat's theorem for stationary points [16], the maximum is reached on the trapeze's boundary, hence, on one of $T$ 's sides.

$P_{d}$ decreases with $a$ along the vertical side $Q P$, so that the maximum on $Q P$ is reached in $Q . P_{d}$ increases with $x$ so that $P_{d}$ increases along sides $Q M$ and $P N$. Therefore, the possible maximum points are on the line segment $S_{M N}$. So all we need to study is the maximum of $P_{d}$ on $M N$. On the segment $M N, x=a u_{0}$, so we can express $P_{d}$ as a function of only one real variable, $a$. Let this function be $\varphi$.

$$
\begin{aligned}
& P_{d}\left(a u_{0}, a\right) \\
& =1-(1-s)\left[1-\frac{Q}{a k}(\sqrt{r}+\sqrt{o})^{2}\right]^{a u_{0}} \\
& \quad-s\left[1-\frac{Q+1}{a k}(\sqrt{r}+\sqrt{o})^{2}\right]^{a u_{0}} \\
& =\varphi(a) .
\end{aligned}
$$


We take the derivative of $\varphi$ and study its sign in order to establish the function's monotony.

$$
\begin{aligned}
& \varphi^{\prime}(a)=-(1-s) u_{0}\left[1-\frac{Q}{a k}(\sqrt{r}+\sqrt{o})^{2}\right]^{a u_{0}} \\
& {\left[\ln \left(1-\frac{Q+1}{a k}+(\sqrt{r}+\sqrt{o})^{2}\right)+a \frac{\left(Q / a^{2} k\right)(\sqrt{r}+\sqrt{o})^{2}}{1-(Q / a k)(\sqrt{r}+\sqrt{o})^{2}}\right]} \\
& -s u_{0}\left[1-\frac{Q}{a k}(\sqrt{r}+\sqrt{o})^{2}\right]^{a u_{0}} \\
& {\left[\ln \left(1-\frac{Q+1}{a k}(\sqrt{r}+\sqrt{o})^{2}\right)+a \frac{\left((Q+1) / a^{2} k\right)(\sqrt{r}+\sqrt{o})^{2}}{1-((Q+1) / a k)(\sqrt{r}+\sqrt{o})^{2}}\right]} \\
& \leq 0,
\end{aligned}
$$

The above is true because the sum of two negative numbers is also negative. Here we have used the inequality:

$$
(1-\rho) \ln (1-\rho)+\rho \geq 0 \text { for all } \rho<1 .
$$

Therefore, $P_{d}$ is non-increasing on $M N$, which means that the maximum is reached at $M: \max P_{d}=P_{d}\left(4 r u_{0}, 4 r\right)$. The monotony of $P_{d}$ along segment $\stackrel{T}{M} N$ also solves the related problem, when $x / a=u_{0}$, as stated in the theorem. We have: $\max _{S_{M N}} P_{d}=P_{d}(M)=P_{d}\left(4 r u_{0}, 4 r\right)$ and $\min _{S_{M N}} P_{d}=P_{d}(N)=$ $P_{d}\left(u_{0} a_{0}, a_{0}\right)$.

Similar computations can be done in the case of a square shaped intrusion object. Hence the results are valid for both square and circular objects.

Proof: (Theorem 3) (a) Direct computations show that $\partial P_{d} / \partial x>0$ and $\partial P_{d} / \partial a<0$. It follows that $x=x(a)$ and $a=a(x)$ are strictly increasing. Let $g=x(a) / a$. A more complicated computation leads to $g^{\prime}(a)>0$ for all $a \in$ $\left[a(4), a_{0}\right]$. More precisely:

$$
\begin{aligned}
& \left(\partial P_{d} / \partial x\right)(x, a) \\
= & -(1-s)\left(1-\frac{o_{1}}{a} \frac{N}{k}\right)^{x} \ln \left(1-\frac{o_{1}}{a} \frac{N}{k}\right) \\
& -s\left(1-\frac{o_{1}}{a} \frac{N+1}{k}\right) \ln \left(1-\frac{o_{1}}{a} \frac{N+1}{k}\right) \\
> & 0,
\end{aligned}
$$

and

$$
\begin{aligned}
& \left(\partial P_{d} / \partial a\right)(x, a) \\
= & -x\left[(1-s)\left(1-\frac{o_{1}}{a} \frac{N}{k}\right)^{x-1} \frac{o_{1}}{a^{2}} \frac{N}{k}\right. \\
& \left.+s\left(1-\frac{o_{1}}{a} \frac{N+1}{k}\right)^{x-1} \frac{o_{1}}{a^{2}} \frac{N+1}{k}\right] \\
< & 0 .
\end{aligned}
$$

Since $u+(1-u) \ln (1-u)>0$ for any $u<1$, we have the following inequality based on the Implicit Function Theorem [17]

$$
\begin{aligned}
& \quad \operatorname{sign}\left(g^{\prime}(a)\right)=\operatorname{sign}\left(x^{\prime}(a) a-x(a)\right) \\
& =\operatorname{sign}\left[-\frac{\partial P_{d} / \partial a}{\partial P_{d} / \partial x}(x(a), a) a-x(a)\right] \\
& =\quad \operatorname{sign}\left(-\frac{\partial P_{d}}{\partial a} a-x(a) \frac{\partial P_{d}}{\partial x}\right) \\
& \operatorname{sign}\left[( 1 - s ) ( 1 - \frac { o _ { 1 } } { a } \frac { N } { k } ) ^ { x - 1 } \left(\frac{o_{1} N}{a k}+\left(1-\frac{o_{1} N}{a k}\right) \ln \left(1-\frac{o_{1} N}{a k}\right)\right.\right. \\
& \left.\left.\quad+s\left(1-\frac{o_{1}(N+1)}{a k}\right) \ln \left(1-\frac{o_{1}(N+1)}{a k}\right)\right)\right]>0 .
\end{aligned}
$$

The conclusion follows.

(b) Consider the expression of $P_{d}$ given by 3 , the first case. This leads immediately to the explicit formulas for $x=x(a)$ and $a=a(x)$ in this particular case. We have: $g(a)=\frac{x(a)}{a}=\frac{\ln \left(1-P_{0}\right)}{a \ln \left(1-o_{1} / a\right)}$. Simple computations show that $g$ is increasing as a function of $a$. Hence $\min (g(a))=\frac{4}{a(4)}=$ $\frac{4}{o_{1}}\left[1-\left(1-P_{0}\right)^{1 / 4}\right]$. This concludes the proof.

Proof: (Lemma 1) $p_{1}(b)=\frac{1}{a}\left[o+2\left(b+\frac{o}{b}\right) \sqrt{\frac{r}{\pi}}+r\right]$. Hence: $p_{1}^{\prime}(b)=\frac{1}{a} 2 \sqrt{\frac{r}{\pi}}\left(1-\frac{o}{b^{2}}\right)\left\{\begin{array}{l}<0, b<\sqrt{o} \\ \geq 0, b \geq \sqrt{o} \text {. Function }\end{array}\right.$ $p_{1}(b)$ first decreases and then increases, so $b_{0}=\sqrt{o}$ is the point for $p_{1}$ to become the minimum.

Proof: (Lemma 2) By using composed function derivation, we have: $\frac{d P_{d}}{d b}=\frac{d P_{d}}{d p_{1}} \frac{d p_{1}}{d b}$. The first fraction on the right hand side is strictly positive, so the sign of $\frac{d P_{d}}{d b}$ is given by the sign of $\frac{d p_{1}}{d b}$, for all $b>0$.

Proof: (Theorem 4)

$$
\begin{aligned}
& P_{d}=1-\left[(1-s)\left(1-\frac{\lceil L / T\rceil}{k} p_{1}\right)^{n}+s\left(1-\frac{\lceil L / T\rceil+1}{k} p_{1}\right)^{n}\right] \\
& <1-(1-s+s)\left(1-\frac{\lceil L / T\rceil+1}{k} p_{1}\right)^{n} \\
& =1-\left(1-\frac{\lceil L / T\rceil+1}{k} p_{1}\right)^{n}
\end{aligned}
$$

Likewise, $P_{d}>1-\left(1-\frac{\lceil L / T]}{k} p_{1}\right)^{n}$.

Proof: (Theorem 5) $V(x, o)-V(2 k, r)=$ $\frac{\partial V}{\partial x}\left(\theta_{1}, \theta_{2}\right)(x-2 k)+\frac{\partial V}{\partial o}\left(\theta_{1}, \theta_{2}\right)(o-r) \geq 0$. The inequality holds since the left side is the sum of two nonnegative terms for any $(x, o) \in S$. Obviously, $x-2 k$ and $o-r$ are nonnegative, so all that remains to be shown is that the first order partial derivatives of $V$ are positive. Recall that for an intrusion object shaped as a disk, the analytic expression of $V$ is: $V(x, o)=1-\left[1-\frac{(\sqrt{r}+\sqrt{o})^{2}}{a}\right]^{x / k} \cdot V$ is an increasing function of both $x$ and $o$, so $\frac{\partial V}{\partial x}>0$ and $\frac{\partial V}{\partial o}>0$. It follows that $V(x, o) \geq V(2 k, r)$ for any $(x, o) \in S$.

Proof: (Corollary 2) By Taylor's formula, we have:

$$
\begin{aligned}
& V(x, o) \\
& =V(2 k, r)+\frac{\partial V}{\partial x}(2 k, r)(x-2 k) \\
& +\frac{\partial V}{\partial o}(2 k, r)(o-r)+\frac{1}{2}\left[\frac{\partial^{2} V}{\partial x^{2}}\left(t_{1}, t_{2}\right)(o-r)^{2}\right. \\
& \left.+2 \frac{\partial^{2} V}{\partial x \partial o}\left(t_{1}, t_{2}\right)(x-2 k)(o-r)+\frac{\partial^{2} V}{\partial o^{2}}\left(t_{1}, t_{2}\right)(o-r)^{2}\right] .
\end{aligned}
$$

The last term is negative, since all second order partial derivatives of $V$ are negative. The conclusion follows from the expression of $V(2 k, r)$ and from the inequality $\ln (1+u) \geq$ $\frac{u}{1+u}, u>-1$.

Proof: (Theorem 6)

$$
\begin{aligned}
& V(x, o)-V\left(n_{0},(\sqrt{a}-\sqrt{r})^{2}\right) \\
= & \frac{\partial V}{\partial x}\left(\theta_{1}, \theta_{2}\right)\left(x-n_{0}\right)+\frac{\partial V}{\partial o}\left(\theta_{1}, \theta_{2}\right)\left(o-(\sqrt{a}-\sqrt{r})^{2}\right),
\end{aligned}
$$

where $\left(\theta_{1}, \theta_{2}\right)$ is a point situated on the segment with end points $(x, o)$ and $\left(n_{0},(\sqrt{a}-\sqrt{r})^{2}\right)$.

The first order partial derivatives of $V(x, o)$ are positive, and are multiplied by negative quantities. Hence, the difference $V(x, o)-V\left(n_{0},(a-\sqrt{r})^{2}\right)$ is negative. Likewise, it can be shown that the difference $V(x, o)-V(2 k, r)$ is positive. The conclusion follows. 
Proof: (Corollary 3)

$$
\begin{aligned}
(1-1 / k)^{n_{0}} & =(1-m / n)^{n_{0}}=e^{n_{0} \ln (1-m / n)} \\
\geq e^{n_{0} \frac{-m / n}{1-m / n}} & =e^{-\frac{m n_{0}}{m-n}} .
\end{aligned}
$$

According to Theorem 6 we can write:

$$
\max _{R_{3}} V=1-(1-1 / k)^{n_{0}} \leq 1-e^{-\frac{m n_{0}}{n-m}} .
$$

For the left-hand side inequality, we use the well-known inequality: $\ln (1+x) \leq x$, for all $x>-1$, and we obtain: $(1-4 r / a k)^{2 k}=e^{2 k \ln (1-4 r / a k)} \leq e^{-\frac{8 r}{a}}$.

Proof: (Corollary 4) According to Corollary 3, we get: $1-e^{-\frac{8 r}{a}}=1-\lim _{k \rightarrow \infty}\left(1-\frac{4 r}{a k}\right)^{2 k} \leq V(x, o) \leq 1-$ $\lim _{n_{0} \rightarrow \infty}\left(1-\frac{m}{n_{0}}\right)^{n_{0}}=1-e^{-m}$.

Proof: (Theorem 7) Consider the expression of $V(x, b)$ given by (6). $\frac{\partial V}{\partial x}(x, b) \geq 0$ for all $(x, b) \in\left[4, n_{0}\right] \times\left[b_{1}, b_{2}\right]$ and $\operatorname{sign}\left(\frac{\partial V}{\partial b}(x, b)\right)=\operatorname{sign}\left(1-\frac{o}{b^{2}}\right)=\left\{\begin{array}{l}1, b>\sqrt{o} \\ 0, b=\sqrt{o} \\ -1, b<\sqrt{o}\end{array}\right.$

Therefore,

$$
\begin{aligned}
& V(x, b) \geq V(x, \sqrt{o})=1-\left[1-\frac{1}{a}\left(o+r+4 \sqrt{\frac{r}{\pi}} \sqrt{o}\right)\right]^{x / k} \\
& \geq 1-\left[1-\frac{1}{a}\left(o+r+4 \sqrt{\frac{r}{\pi}} \sqrt{o}\right)\right]^{4 / k} .
\end{aligned}
$$

On the other hand, from the signature of $V$ we know that $V$ decreases and then increases with respect to $b$. Therefore the maximum is reached in one of the extremities $b_{1}, b_{2}$. We have: $V(x, b)$

$$
\begin{aligned}
& \leq \max _{j \in\{1,2\}}\left\{1-\left(1-\frac{o+r+\sqrt{r / \pi}\left(b_{j}+o / b_{j}\right)}{a}\right)^{x / k}\right\} \\
& \leq \max _{j \in\{1,2\}}\left\{1-\left(1-\frac{o+r+\sqrt{r / \pi}\left(b_{j}+o / b_{j}\right)}{a}\right)^{n_{0} / k}\right\} .
\end{aligned}
$$

Proof: (Theorem 8) From Section IV we know that $V_{n}=$ $1-\left[1-p_{1}\right]^{n / k}$. We use the following well-known inequality: $\left(1-\frac{\alpha}{n}\right)^{n} \leq e^{-\alpha}, \quad \alpha \geq 0 . V_{n}$ is therefore bounded by:

$$
V_{n} \geq 1-e^{-m p_{1}} \Rightarrow 1-V_{x} \leq e^{-m p_{1}} .
$$

On the other hand, it is well known that

$$
V_{n}\left(1-V_{n}\right) \leq \frac{1}{4}
$$

It follows that: $1-V_{n} \leq \frac{1}{4 V_{n}} \stackrel{(9)}{\leq} \frac{1}{4\left(1-e^{\left.-m p_{1}\right)}\right.}$.

$$
\Rightarrow V_{n} \geq 1-\frac{1}{4\left(1-e^{-m p_{1}}\right)}=\frac{3-4 e^{-p_{1} m}}{4\left(1-e^{-p_{1} m}\right)} .
$$

From (9) and (11) we obtain the conclusion: $V_{n} \geq$ $\max \left\{1-e^{-p_{1} m}, \frac{3-4 e^{-p_{1} m}}{4\left(1-e^{-p_{1} m}\right)}\right\}$.

Following, we prove the upper bound. Using inequality: $\ln (1+x) \geq \frac{x}{x+1}$, for all $x>-1$, we obtain: $\frac{n}{k} \ln \left(1-p_{1}\right) \geq$ $-\frac{m p_{1}}{1-p_{1}}$. It follows that: $\left(1-p_{1}\right)^{n / k} \geq e^{-\frac{m p_{1}}{1-p_{1}}}$. This leads to the conclusion $V_{n} \leq 1-e^{-\frac{m p_{1}}{1-p_{1}}}$.

Proof: (Theorem 9) In the case of a circular intrusion object, by combining Equations (1) and (3), $P_{d}$ is given by:

$$
\begin{aligned}
P_{d}=1 & -(1-s)\left[1-\frac{Q}{a k}(\sqrt{r}+\sqrt{o})^{2}\right]^{c} \\
& -s\left[1-\frac{Q+1}{a k}(\sqrt{r}+\sqrt{o})^{2}\right]^{c} .
\end{aligned}
$$

We have

$\frac{\partial P_{d}}{\partial o}=\frac{c}{a k}(1+\sqrt{r / o})\left[(1-s) Q\left(1-\frac{Q}{a k}(\sqrt{r}+\sqrt{o})^{2}\right)^{c-1}+\right.$ $\left.s(Q+1)\left(1-\frac{Q+1}{a k}(\sqrt{r}+\sqrt{o})^{2}\right)^{c-1}\right]>0$

$\frac{\partial P_{d}}{\partial k}=-\frac{c}{a k^{2}}(\sqrt{r}+\sqrt{o})^{2}\left[(1-s) Q\left(1-\frac{Q}{a k}(\sqrt{r}+\sqrt{o})^{2}\right)^{c-1}+\right.$ $\left.s(Q+1)\left(1-\frac{Q+1}{a k}(\sqrt{r}+\sqrt{o})^{2}\right)^{c-1}\right]<0$

Therefore, $\partial P_{d} / \partial o>0$ and $\partial P_{d} / \partial k<0$.

According to the Implicit Function Theorem [17], around each point $(o, k)$ which satisfies the constraints in the theorem's hypothesis and the fundamental equality $P_{d}\left(o^{\prime}, k\right)=$ $P_{0}$, there exists a function $k=k\left(o^{\prime}\right)$ having derivatives of any order, such that $P_{d}\left(o^{\prime}, k\left(o^{\prime}\right)\right)=P_{0}$ for any $o^{\prime}$ close to $o$.

The first order derivative of $k$ is given by: $k^{\prime}(o)=$ $-\frac{\frac{\partial P_{d}}{\partial o}(o, k(o))}{\frac{\partial P_{d}}{\partial k}(o, k(o))}=\frac{k(o)}{\sqrt{r} \sqrt{o}+o}>0$.

Dividing by $k(o)$ and integrating, we get: $\int_{o_{1}}^{o} \frac{k^{\prime}(t)}{k(t)} d t=$ $\int_{o_{1}}^{o} \frac{d t}{t+\sqrt{r} \sqrt{t}} \stackrel{\sqrt{t}=u}{=} \int_{\sqrt{o_{1}}}^{\sqrt{o}} \frac{2 d u}{u+\sqrt{r}}=2 \ln \frac{\sqrt{o}+\sqrt{r}}{\sqrt{o_{1}}+\sqrt{r}}$.

This implies:

$$
\frac{k(o)}{k\left(o_{1}\right)}=\left(\frac{\sqrt{o}+\sqrt{r}}{\sqrt{o_{1}}+\sqrt{r}}\right)^{2} .
$$

Using condition $(d$.$) and using the fact that k$ is an increasing function of $o$, we obtain the constrained solution: $\max k=\left\lfloor k\left((\sqrt{a}-\sqrt{r})^{2}\right)\right\rfloor=\left\lfloor\frac{a}{\left(\sqrt{o_{1}}+\sqrt{r}\right)^{2}}\right\rfloor$.

In addition, from $\left(c\right.$. ) we have that $k\left(o_{1}\right)=1$. Therefore, using (10) we obtain $k(o)=\left(\frac{\sqrt{o}+\sqrt{r}}{\sqrt{o_{1}}+\sqrt{r}}\right)^{2}$.

Proof: (Theorem 10) By combining Equations (2) and (3), we have:

$$
\begin{aligned}
& P_{d}=1-(1-s)\left[1-\frac{Q}{a k}(o+2 \sqrt{r / \pi}(b+o / b)+r)\right]^{c} \\
& -s\left[1-\frac{Q+1}{a k}(o+2 \sqrt{r / \pi}(b+o / b)+r)\right]^{c} . \\
& \frac{\partial P_{d}}{\partial b}=\frac{2 c}{a k} \sqrt{\frac{r}{\pi}}\left(1-\frac{o}{b^{2}}\right)\left[( 1 - s ) Q \left(1-\frac{Q}{a k}\right.\right. \\
& (o+2 \sqrt{r / \pi}(b+o / b)+r))^{c-1}+s(Q+1) \\
& \left.\left(1-\frac{Q+1}{a k}(o+2 \sqrt{r / \pi}(b+o / b)+r)\right)^{c-1}\right] . \\
& \frac{\partial P_{d}}{\partial k}=-\frac{c[o+2 \sqrt{r / \pi}(b+o / b)+r]}{a k^{2}}\left[( 1 - s ) Q \left(1-\frac{Q}{a k}\right.\right. \\
& (o+2 \sqrt{r / \pi}(b+o / b)+r))^{c-1}+s(Q+1) \\
& \left.\left(1-\frac{Q+1}{a k}(o+2 \sqrt{r / \pi}(b+o / b)+r)\right)^{c-1}\right]<0 .
\end{aligned}
$$

Next, we apply the Implicit Function Theorem [17]. We have: $k^{\prime}(b)=-\frac{\frac{\partial P_{d}}{\partial P_{d}}(b, k(b))}{\frac{\partial P^{d}}{\partial k}(b, k(b))}=k(b) \frac{2 \sqrt{r / \pi}\left(1-o / b^{2}\right)}{o+2 \sqrt{r / \pi}(b+o / b)+r}$.

This implies: $\int_{b_{1}}^{b} \frac{k^{\prime}(t)}{k(t)} d t=\ln \frac{o+2 \sqrt{r / \pi}(b+o / b)+r}{o+2 \sqrt{r / \pi}\left(b_{1}+o / b_{1}\right)+r} \Rightarrow$ $k(b)=\frac{o+2 \sqrt{r / \pi}(b+o / b)+r}{o+2 \sqrt{r / \pi}\left(b_{1}+o / b_{1}\right)+r}$.

The last equation, the signature of $k^{\prime}(b)$, and the fact that $b_{1}+o / b_{1} \leq b_{2}+o / b_{2}$ together imply:

$\max k=\left\lfloor k\left(b_{2}\right)\right\rfloor=\left\lfloor a /\left(o+2 \sqrt{r / \pi}\left(b_{1}+o / b_{1}\right)+r\right)\right\rfloor$.

\section{REFERENCES}

[1] Z. Abrams, A. Goel, and S. Plotkin, "Set k-cover algorithms for energy efficient monitoring in WSNs," in Proc. IPSN 2004. 
[2] C. Hsin and M. Liu, "Network coverage using low duty-cycled sensors: random and coordinated sleep algorithm," in Proc. IPSN 2004.

[3] S. Meguerdichian, F. Koushanfar, M. Potkonjak, and M. Srivastava, "Coverage problems in wireless ad-hoc sensor networks," in Proc. IEEE INFOCOM 2001.

[4] S. Ren, Q. Li, H. Wang, X. Chen, and X. Zhang, "Design and analysis of sensing scheduling algorithms under partial coverage for object detection in sensor networks," IEEE Trans. Parallel Distrib. Syst., vol. 18, no. 3, pp. 334-350, Mar. 2007.

[5] D. Tian and D. Georganas, "A coverage-preserving node scheduling scheme for large WSNs," in Proc. WSNA 2002.

[6] K. Wu, Y. Gao, F. Li, and Y. Xiao, "Lightweight deployment-aware scheduling for WSNs," Mobile Networks and Applications, vol. 10, no. 6, pp. 837-852, Dec. 2005.

[7] T. Yan, T. He, and J. Stankovic, "Differentiated surveillance for sensor networks," in Proc. ACM SenSys 2003.

[8] F. Ye, G. Zhong, J. Cheng, S. Lu, and L. Zhang, "Peas: a robust energy conserving protocol for long-lived sensor networks," in Proc. ICNP 2002.

[9] L. Wang and Y. Xiao, "A survey of energy-efficient scheduling mechanisms in sensor networks," Mobile Networks and Applications, vol. 11, no. 5, pp. 723-740, Oct. 2006.

[10] C. Liu, K. Wu, Y. Xiao, and B. Sun, "Random coverage with guaranteed connectivity: joint scheduling for WSNs," IEEE Trans. Parallel Distrib. Syst., vol. 17, no. 6, pp. 562-575, June 2006.

[11] Y. Xiao, H. Chen, K. Wu, C. Liu, and B. Sun, "Maximizing network lifetime under QoS constraints in WSNs," in Proc. GLOBECOM 2006.

[12] Y. Xiao, H. Chen, K. Wu, and B. Sun, "Modeling detection metrics in randomized scheduling algorithm in wireless sensor networks," in Proc. IEEE WCNC 2007.

[13] Y. Xiao, Y. Zhang, X. Sun, and H. Chen, "Asymptotic coverage and detection in randomized scheduling algorithm in wireless sensor networks," in Proc. IEEE ICC 2007.

[14] Y. Xiao, H. Chen, Y. Zhang, X. Du, B. Sun, and K. Wu, "Intrusion objects with shapes under randomized scheduling algorithm in sensor networks," in Proc. IEEE ICDCS Workshops.

[15] P. K. Sahoo and T. Riedel, Mean Value Theorems and Functional Equations. World Scientific Publisher Company, 1999.

[16] J. Brinkhuis and V. Tikhomirov, Optimization: Insights and Applications. Princeton University Press, 2005.

[17] L. D. Kudryavtsev, "Implicit function," in Encyclopedia of Mathematics, M. Hazewinkel, ed. Kluwer, 1990.

[18] Y. Xiao, H. Chen, K. Wu, B. Sun, Y. Zhang, X. Sun, and C. Liu, "Coverage and detection of a randomized scheduling algorithm in wireless sensor networks," IEEE Trans. Computers, accepted. DOI: 10.1109/TC.2009.170

[19] Y. Xiao, Y. Zhang, M. Peng, H. Chen, X. Du, B. Sun, and K. Wu, "Two and Three-Dimensional Intrusion Object Detection under Randomized

Scheduling Algorithms in Sensor Networks," Computer Networks, Vol. 53, No. 14 , Sept. 2009 , pp. 2458-2475.
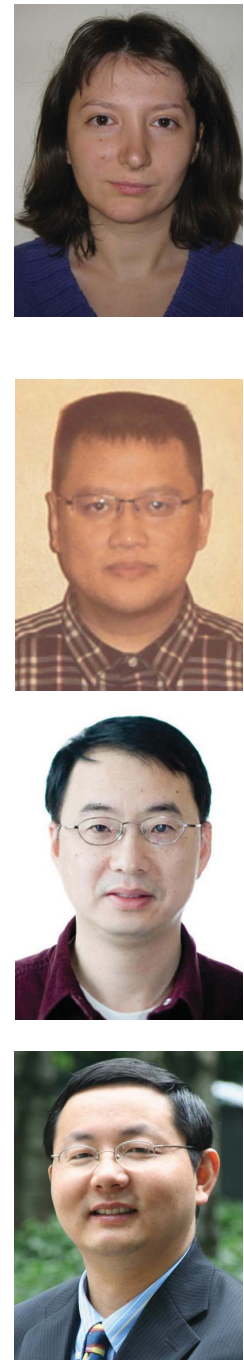

Alina Olteanu received her B.S. degree in Computer Science and her M.S. degree in Applied Mathematics from the University of Bucharest and Polytechnic University of Bucharest, Romania in 2003 and 2005, respectively, and earned her Ph.D. degree in Computer Science from the University of Alabama, USA in 2009. Her research interests are in the areas of wireless network security, network performance optimization and lightweight cryptography.

Yang Xiao (SM04) is currently with Department of Computer Science, the University of Alabama, USA. He currently serves as Editor-in-Chief for INTERNATIONAL JOURNAL OF SECURITY AND NETWORKS and INTERNATIONAL JOURNAL OF SENSOR NETWORKS. His research interests are security, telemedicine, robots, and sensor/wireless networks. He was a voting member of the IEEE 802.11 Working Group from 2001 to 2004.

Kui Wu received the Ph.D. degree in Computing Science from the University of Alberta, Canada, in 2002, and joined the Department of Computer Science, University of Victoria, Canada in the same year, where he is currently an Associate Professor. His research interests include performance analysis and protocol design of computer networks, wireless sensor networks, and network security. $\mathrm{He}$ is an IEEE senior member.

Xiaojiang Du is an Assistant Professor in the Dept. of Computer and Information Sciences at Temple Univ. He was an Assistant Professor in the Dept. of Computer Science at North Dakota State Univ. between Aug. 2004 and Jun. 2009, where he received the Excellence in Research Award in May 2009. Dr. Du's research interests are wireless networks, security, computer networks and systems. He has published over 70 journal and conference papers and has been awarded more than $\$ 1 M$ research grants from the U.S. NSF and Army Research Office. 